\title{
e-Migrinter
}

$5 \mid 2010$

Migrations dans les pays nordiques

\section{Liégeois, Jean-Pierre (2009) Roms et Tsiganes}

\section{Céline Bergeon}

\section{(2) OpenEdition}

Journals

Édition électronique

URL : https://journals.openedition.org/e-migrinter/1931

DOI : 10.4000/e-migrinter.1931

ISSN : 1961-9685

\section{Éditeur}

UMR 7301 - Migrinter

\section{Édition imprimée}

Date de publication : 28 avril 2010

Pagination : 76-77

ISSN : 1961-9685

\section{Référence électronique}

Céline Bergeon, «Liégeois, Jean-Pierre (2009) Roms et Tsiganes », e-Migrinter [En ligne], 5 | 2010, mis en ligne le 20 avril 2020, consulté le 20 mai 2021. URL : http://journals.openedition.org/e-migrinter/ 1931 ; DOI : https://doi.org/10.4000/e-migrinter.1931 


\section{Liégeois, Jean-Pierre (2009) Roms et Tsiganes, Paris, La Découverte, 125 p.}

\section{Céline Bergeon}

C et ouvrage de Jean-Pierre Liégeois vient enrichir la bibliographie spécifique de l'auteur sur la population des Roms et des Tsiganes. C'est une synthèse descriptive qui s'attache particulièrement à définir l'organisation sociale de cette population au regard des environnements sociopolitiques dans lesquels elle évolue. C'est donc avec une vision dynamique que l'auteur appréhende ces acteurs. Jean-Pierre Liégeois insiste avec minutie sur le rapport qu'entretiennent les Roms et les Tsiganes avec les sociétés d'accueil. Comment les acquis culturels sont-ils mobilisés et tendentils à l'évolution dans les contextes actuels ?

La première s'intitule «images et réalités » comme pour décrire une habituelle distance entre ce qui est observé par ceux de «l'extérieur» et ce qui est réellement vécu par les Tsiganes. L'auteur décrit le processus qui amène la supposition, l'image, supposée comme certitude, à s'introduire dans le champ de la discrimination et de la négation. De par leurs origines troubles et leurs habitudes de vie, les Tsiganes interpellent les sociétés sédentaires bien que la majeure partie d'entre eux ne pratique plus qu'une mobilité réduite. C'est donc par un court rappel historique que Jean-Pierre Liégeois nous mène sur les chemins des migrations Tsiganes jusqu'à leur arrivée en Europe occidentale. Par ailleurs, il insiste sur la problématique nominative, qui pour lui, est un «grand malentendu». Le panel des appellations est important. Il montre la difficile appréhension de ces populations au sein des espaces nationaux dans le même temps qu'il impulse des connotations péjoratives: les termes employés par l'extérieur ont acquis des connotations péjoratives dans la plupart des langues, l'expression gens du voyage permet de désigner de façon globalisante et vague sans la nommer une réalité etbno-culturelle que l'État refuse de reconnaître (p. 27). Ainsi les dénominations viennent appuyer des stéréotypes ancrés historiquement dans les mentalités, introduisant trop souvent des situations discriminantes.

La deuxième partie de l'ouvrage s'intitule «une négation séculaire »; l'auteur y décrit avec pertinence les phases par lesquelles sont passées les populations tsiganes dans les rapports qu'elles entretiennent avec la société. Exclusion, réclusion, inclusion en passant par l'extermination, sont donc pour Jean-Pierre Liégeois les principales modalités d'appréhension des populations tsiganes que les sociétés sédentaires ont développé. L'analyse des politiques a notamment permis à l'auteur de comprendre la nature des traitements infligés aux Tsiganes unanimement liés par le rejet (p. 43). L'auteur porte une attention particulière à l'exemple de la France, qui a instauré de nombreuses législations spécifiques afin de maitriser cette population. L'exclusion en tant que telle produit du rejet et entretient la crainte, elle s'efforce de repousser hors des frontières ces populations problématiques. Néanmoins, devant l'inefficacité de ces procédures, la réclusion fait son apparition dans les discours et les attitudes des pouvoirs publics (l'auteur précise ici qu'au niveau local, à l'échelle de l'action, l'exclusion reste toutefois le maitre mot). Les autorités sont donc passées d'une logique d'exclusion à 
celle de l'intégration, qualifiée d'autoritaire et de violente par Jean-Pierre Liégeois. Au $17^{\text {ème }}$ siècle en Autriche, les Tsiganes surpris à parler leur langue étaient punis de 25 coups de bâton (p. 52). Enfin, les élans humanistes du début des années 1970 ont amené les législateurs et les autorités politiques à revisiter leurs modes d'action en considérant le tsigane comme une personne "marginale » à réinsérer alors que le reclus est puni, l'inclus est récompensé de sa normalisation (p. 57). L'ensemble de ces dispositions ont eu de réels impacts sur la prise en charge des populations tsiganes à l'échelle locale.

La dernière partie de cet ouvrage, «la politique des roms", introduit un changement dans l'étude de cette population. En effet, au même titre que les populations sédentaires, ces groupes sont également touchés par les mutations socioéconomiques qui animent aujourd'hui les sociétés. C'est par conséquent dans un contexte dynamique de changement que les populations tsiganes redéfinissent leur position au sein des institutions, alors que ces mêmes évolutions socio-économiques renforcent des sentiments d'incompréhension et de rejet émanant de la société d'accueil. Ainsi, afin de décrire au lecteur de façon la plus pertinente possible les adaptations et les mutations à l'intérieur des communautés tsiganes, Jean-Pierre Liégeois revient sur ce qui fonde le quotidien et le mode de vie de ces populations: comprendre ce qui est, pour mieux saisir ce qui est en changement. En terminant son ouvrage par l'implication des organismes internationaux dans la reconnaissance de ces populations, l'auteur s'autorise à suggérer une éventuelle amélioration, ou tout au moins une évolution du poids des instances tsiganes dans les représentations internationales, ce qui impliquerait une meilleure acceptation de cette population.
De par la qualité des connaissances de Jean-Pierre Liégeois sur les tsiganes, cet ouvrage représente une synthèse intéressante sur les spécificités roms, remises à jour par leur mise en relation étroite avec les évolutions socio-économiques des sociétés d'accueil. L'auteur offre également des repères bibliographiques pertinents sur la population tsigane.
Céline Bergeon Doctorante en géographie MIGRINTER - UMR 6588 CNRS / Université de Poitiers bergeonceline@yahoo.fr 